\title{
Terms and Definitions Used to Describe Recurrence, Treatment Failure and Recovery of Acute Exacerbations of COPD: A Systematic Review of Observational Studies
}

\author{
Wilhelmine $\mathrm{H}$ Meeraus' \\ Bailey M DeBarmore ${ }^{2}$ \\ Hana Mullerova (iD) \\ William A Fahy ${ }^{3}$ \\ Victoria S Benson (D) \\ 'Epidemiology - Value Evidence and \\ Outcomes, Global Medical R\&D, \\ GlaxoSmithKline, Brentford, UK; \\ ${ }^{2}$ Epidemiology - Value Evidence and \\ Outcomes, Global Medical R\&D, \\ GlaxoSmithKline, Raleigh, NC, USA; \\ ${ }^{3}$ Discovery Medicine, Research and \\ Development, GlaxoSmithKline, \\ Stevenage, UK
}

\begin{abstract}
Introduction: Acute exacerbations of chronic obstructive pulmonary disease (AECOPDs) are important clinical events, with many patients experiencing multiple AECOPDs annually. The terms used in the literature to define recurring AECOPD events are inconsistent and may impact the ability to describe the true burden of these events. We undertook a systematic review to identify and summarize terms and definitions used in observational studies to describe AECOPD-related events occurring after an initial AECOPD (hereafter "subsequent AECOPD"). Methods: PubMed was searched (2000-2019) for observational studies on subsequent AECOPD events using broad search strings for "COPD", "exacerbation", and "subsequent exacerbation events". Only English-language studies were included. Small studies $(\mathrm{n}<50)$ and studies focusing on hospital re-admission only were excluded. Extracted data were analyzed descriptively to generate a narrative summary, using a thematic approach to group studies utilizing similar terms for subsequent AECOPD.

Results: Forty-seven studies were included. No single, distinct terms or definitions were used to define and identify multiple occurrences of AECOPDs, though most (46) studies used one or more of four clustered terms and definitions: reapse $(n=13)$, recurrence/re-exacerbation $(n=11)$, treatment failure $(n=12)$ and non-recovery/time to recovery $(n=16)$. Heterogeneity was observed within and between the four clusters with respect to study setting, starting point for observing subsequent AECOPDs, time frame to identify a subsequent AECOPD (except for studies using "time to recovery"), and basis for identifying a subsequent exacerbation.

Conclusion: Our review demonstrates that subsequent AECOPDs (including events such as relapse, recurrence/re-exacerbation, treatment failure, non-recovery/time to recovery) are illdefined in the observational study literature, emphasizing the need to reach consensus on precise and objective definitions (for example, when one AECOPD ends and another begins). Use of standardized terminology and definitions may aid comparability between, and synthesis of, studies, thus improving the understanding of the natural history and burden of exacerbations in COPD patients.
\end{abstract}

Keywords: chronic obstructive pulmonary disease, recurrence, exacerbation, review

\section{Introduction}

Chronic obstructive pulmonary disease (COPD) is the 3rd leading cause of mortality worldwide ${ }^{1}$ and a significant cause of morbidity. ${ }^{2}$ Acute exacerbations of COPD (AECOPDs) are important clinical events and contribute significantly to overall
Correspondence: Victoria S Benson Epidemiology, Value Evidence and Outcomes, Global Medical R\&D, GlaxoSmithKline, 980 Great West Road, Brentford, Middlesex, TW8 9GS, UK Email Victoria.x.tribble@gsk.com 
disease burden and mortality. ${ }^{3}$ AECOPDs are generally defined as an acute, and sustained, worsening of respiratory symptoms requiring additional treatment, including hospitalization when severe. ${ }^{4-6}$ Approximately $50 \%$ of COPD patients experience at least one AECOPD event each year, with exacerbations occurring across all stages of disease severity. ${ }^{7,8}$ Frequent AECOPD events may increase a patient's risk of mortality, ${ }^{9}$ and decrease their lung function ${ }^{10-12}$ and quality of life. ${ }^{13}$ Severe AECOPDs pose a financial burden, with AECOPD hospitalizations accounting for up to $70 \%$ of COPD-related healthcare expenditures. $^{14-16}$

The European Respiratory Society/American Thoracic Society guidelines on COPD management of exacerbations proposed in 2017 state that exacerbations are defined as "episodes of increasing respiratory symptoms, particularly dyspnea, cough and sputum production, and increased sputum purulence". ${ }^{17}$ In clinical trials and observational studies, AECOPDs tend to be defined based on symptoms (eg, following the classic Anthonisen classification), ${ }^{18}$ healthcare utilization (eg, prescriptions for AECOPD treatment, management in hospital), or both. ${ }^{19}$ Identification of symptoms suggestive of AECOPDs may be prospectively recorded in patient daily diaries, ${ }^{20}$ or retrospectively recalled in patient interviews. Alternatively, evidence of healthcare resource utilization (HCRU) for AECOPD may be identified using records in an electronic health records or administrative claims database. A prospective analysis of exacerbations identified by either symptoms or HCRU demonstrated a higher mean rate of exacerbations using the HCRU definition and showed limited agreement between HCRU and symptom-based exacerbations, thus complicating the evaluation of exacerbations in published studies. ${ }^{21}$

Despite the existence of guidelines and efforts to clarify best practice, there is no international consensus or standardized definition of how the start and end dates for exacerbations should be defined. For example, discharge from hospital for treatment of an AECOPD is often used to define the end of an exacerbation in observational studies; however, patients are still likely to be experiencing exacerbation symptoms after discharge, with exacerbation symptoms typically lasting around a week and some lasting as long as 8 weeks or more. ${ }^{22}$ Whilst algorithms have been defined and validated to identify AECOPD in electronic health records, ${ }^{23}$ there remains, however, considerable heterogeneity amongst the definitions for AECOPD. ${ }^{19}$
In addition to challenges that exist with defining when an AECOPD event starts and ends, there are further challenges in defining recurring AECOPD events (ie, when does the initial AECOPD event end and another start), as well as AECOPD recovery and treatment failure. Collectively, we refer to these AECOPD-related events occurring after an initial AECOPD as "subsequent AECOPD events". Differences in study designs, definitions, and how AECOPD was ascertained yield different estimates of re-exacerbation risk within discrete time periods. There is recognition within the research community that a lack of standardized, consistently used definition for exacerbations hampers efforts to assess new therapeutic approaches for AECOPD treatment. ${ }^{24,25}$ As a first step in obtaining external consensus on how subsequent mild, moderate or severe AECOPD events should be defined, our primary objective was to conduct a systematic review to identify and critically summarize the terms and definitions used in observational studies to describe recurrence, treatment failure and recovery of AECOPD. In order to demonstrate the impact on potential inconsistency of definitions for subsequent AECOPD events, a secondary objective was to report the frequency of recurrent AECOPD events, in addition to non-recovery and treatment failure events.

\section{Methods}

We performed a systematic review of observational studies reported in the literature. PubMed was searched for relevant studies published between 1 January 2000 and 31 December 2019. Search strings for terms relating to "disease", "exacerbation", and "subsequent exacerbation events" were combined using an AND operator (see Supplement for details). The search was limited to articles with available abstracts and published in the English language; there was no restriction on the geographical scope.

Articles describing subsequent AECOPD events (eg, recurrence, treatment failure or recovery) in relation to an initial or index AECOPD event were included, with screening performed in two stages. In Stage 1, titles and abstracts were reviewed for the following exclusion criteria: study not presenting original observational research (clinical trials, randomized clinical trials, narrative reviews, letters, case reports); study reporting hospital readmission only; no outcomes of interest (ie, subsequent AECOPD); articles did not answer review objective. In Stage 2, the full text of articles identified in Stage 1 for potential inclusion were reviewed for all the exclusion 
criteria from Stage 1, plus the following additional exclusion criteria: study not describing subsequent AECOPD events in relation to an index AECOPD, no outcome of interest, hospital readmission only study including chronic bronchitis patients only, sample size $<50$, review article. Disagreements were resolved at both stages by consensus or arbitration with an additional reviewer. As a minimum, 1 person screened the titles/abstracts and performed the full text selection, with another extracting the data. Each step was cross-checked by an independent reviewer. Study investigators were not contacted.

Data from the included studies were extracted into evidence tables summarizing study design and population, definition and terminology for subsequent AECOPD event(s), time point from which subsequent AECOPD events were observed (eg, hospital discharge), and length of follow-up to observe subsequent AECOPD events. Where available, the proportion of patients experiencing a subsequent AECOPD event, or the mean time to subsequent AECOPD event was extracted. Data extraction was cross-checked by multiple researchers. Quality of the included studies was assessed broadly with a focus on the completeness of the subsequent AECOPD definition. No studies were excluded from the review based on quality assessment. Extracted data were analyzed descriptively to generate a narrative summary. A thematic approach was used to group studies utilizing similar terms for subsequent AECOPD.

This comprehensive review followed the 2020 Preferred Reporting Items for Systematic Reviews and Meta-Analyses guidelines ${ }^{26}$ for systematic reviews except for two points: the review was not registered and no formal assessment of the risk of bias or quality of the evidence for included studies was performed, however, this was informally assessed.

\section{Results}

At Stage 1 (title and abstract review), 1291 articles (of 1427 identified) were excluded, with 136 articles proceeding to Stage 2 screening (full text review). At Stage 2, 89 articles were excluded, leaving 47 selected for data extraction. The number of articles identified, screened, assessed for eligibility and then included in the review are presented in Figure 1. A description of 46 of the included articles is in Tables 1-4 with further description of all 47 articles in the Supplement.

\section{Terminology}

Terminology describing subsequent AECOPD was clustered into four broad groups: relapse, recurrence/reexacerbation, treatment failure, non-recovery/time to recovery (Tables 1-5). The majority (46) of included studies used these terms and definitions, with some studies using two or more of the four terminology groups. , 27,28 $^{8}$ A single study (Matkovic [2012]) used the term adverse outcome, which had a definition similar to definitions for treatment failure in other studies, but was not grouped with the other studies on the basis that the term differed from the four common terms. ${ }^{29}$

\section{Relapse}

Exacerbation relapse was a term used by 13 articles and was typically defined as a return to the emergency department (ED) or another physician visit for worsening symptoms and typically not differentiated from recurrence or re-exacerbation (Table 1). ${ }^{27,28,30-40}$ Only relapses of moderate or severe exacerbations were reported. The most common study setting where the term relapse was used was the $\operatorname{ED}(n=6)$, followed by outpatient clinics $(n=3)$, hospital $(n=2)$, and then primary care $(n=1)$. One study was set in both the hospital and ED. ${ }^{38}$ Almost all studies calculated the proportion of patients with relapse using the number of patients as the denominator: four studies used exacerbations or patient visits as the denominator. $^{28,31,32,40}$ The timeframe for observing relapse events ranged from five days to one month, with fourteen days being the most common (Figure 2A). The proportion of patients with exacerbation relapse during the timeframe ranged from $7.4 \%$ at/before 20 days to $34 \%$ at/before one month with no clear trend observed over time.

\section{Recurrence/Re-Exacerbation}

The terms exacerbation recurrence or re-exacerbation were used in 11 articles (Table 2). ${ }^{28,41-50}$ Most studies defined recurrence or re-exacerbation as a worsening of symptoms $(n=3)$, subsequent prescription of oral corticosteroids or antibiotics $(\mathrm{n}=2)$, or as a composite outcome including treatment or readmission for AECOPD following an initial AECOPD $(n=3)$. Only four studies specified that recovery from initial AECOPD was required prior to subsequent AECOPD and only recurrence/re-exacerbation of moderate or severe exacerbations were reported. The most common study setting was the hospital $(n=7)$, followed by outpatient clinics $(n=2)$, and primary care $(n=1)$. One study included both hospital inpatient and outpatient 


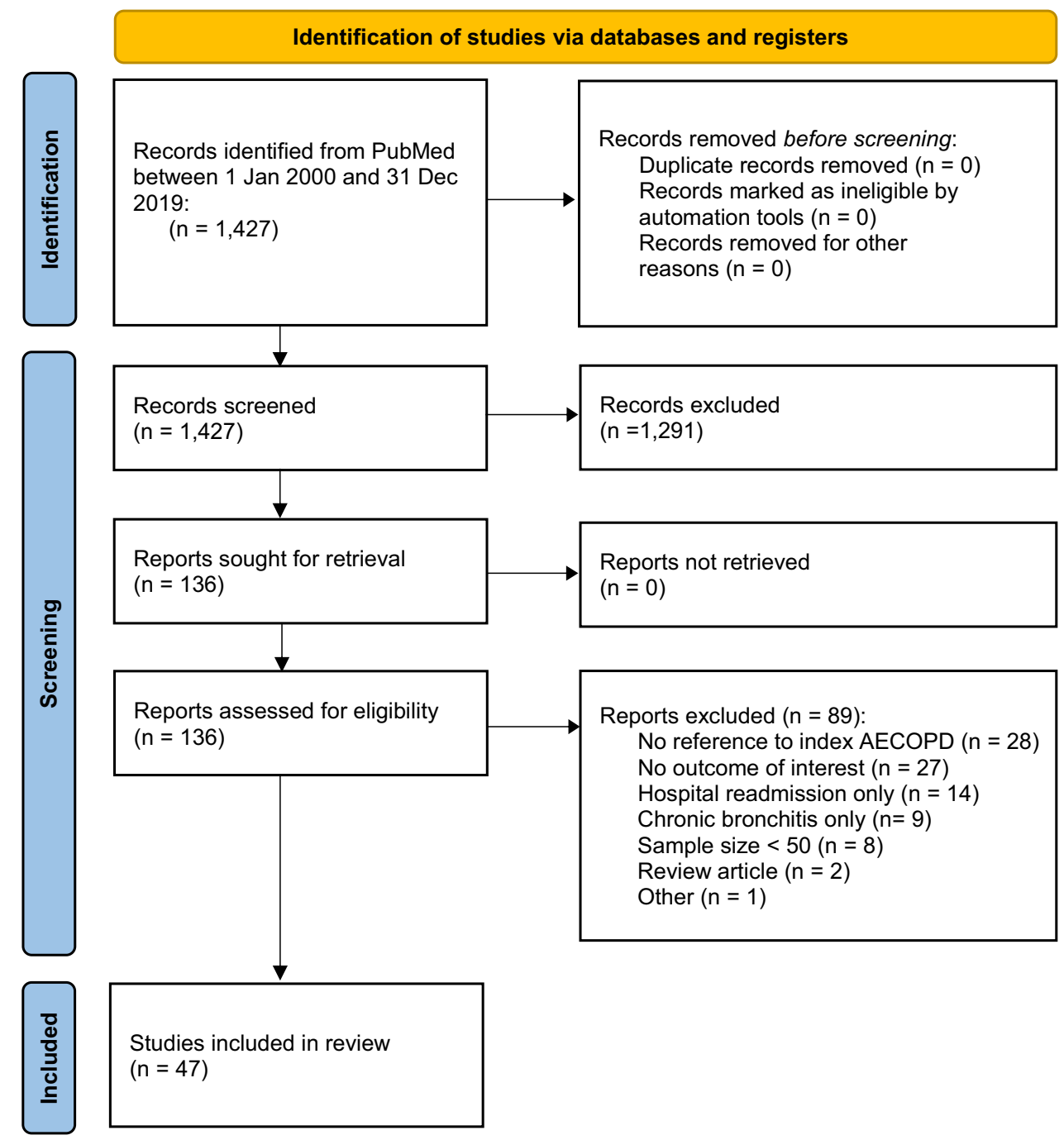

Figure I PRISMA* flow diagram of included and excluded articles. *Page MJ, McKenzie JE, Bossuyt PM, et al. The PRISMA 2020 statement: an updated guideline for reporting systematic reviews. BMJ 2021;372:n7I. doi: I0.1 I36/bmj.n7I. ${ }^{26}$

Abbreviations: AECOPD, acute exacerbation of chronic obstructive pulmonary disease; PRISMA, The Preferred Reporting Items for Systematic reviews and MetaAnalyses.

participants. ${ }^{50}$ The timeframe for recurrence or reexacerbation definitions reporting proportions ranged from one month to 12 months, with either hospital discharge as the initial time point for follow-up (which may or may not be the point at which a patient has "recovered") $(n=6)$ or measured following consecutive days free of recorded symptoms $(n=1)$. The proportion of patients with recurrence/re-exacerbation ranged from $7.4 \%$ at/ before one month to $88.8 \%$ at/before one year, in the same study. Figure 2B shows a trend between length of follow-up and the proportion of patients with a recurrence/ re-exacerbation event; however, variability is still seen among studies using the same starting point for followup (eg, by 90 days, the proportion of patients with an event following hospital discharge ranged from $25 \%$ to $49 \%$ ).

\section{Treatment Failure}

Treatment failure was a term used by 12 articles and was defined as a composite of absence of symptom resolution $(\mathrm{n}=2)$, antibiotic or oral corticosteroid prescription or change in medication $(\mathrm{n}=3)$, hospital readmission $(n=7)$, increased mechanical ventilator support $(\mathrm{n}=5)$, and/or death $(\mathrm{n}=7) .{ }^{51-62}$ One study defined treatment failure as failure to return to baseline or need for a new treatment medication. ${ }^{57}$ Treatment failure was a term only used for moderate or severe exacerbations. The most common study setting for treatment failure was the hospital $(\mathrm{n}=7)$, with other studies set in outpatient clinics $(n=3)$, primary care $(n=1)$, and home care $(n=1)$. The timeframe for observing treatment failure ranged from two days from hospital 
Table I Characteristics of Studies Observing Relapse Events Following an Exacerbation

\begin{tabular}{|c|c|c|c|c|c|c|}
\hline $\begin{array}{l}\text { Author, } \\
\text { Year }\end{array}$ & Relapse Definition & $\mathbf{N}^{\mathbf{a}}$ & $\begin{array}{l}\text { Country, } \\
\text { Study }\end{array}$ & $\begin{array}{l}\text { Study } \\
\text { Setting }\end{array}$ & $\begin{array}{l}\text { Time Point From } \\
\text { Which Relapse is } \\
\text { Measured }\end{array}$ & $\begin{array}{l}\text { Proportion } \\
\text { Relapsed [Time } \\
\text { Frame, (Days)] }^{\mathrm{b}}\end{array}$ \\
\hline $\begin{array}{l}\text { Aaron } \\
2002^{30}\end{array}$ & $\begin{array}{l}\text { Re-visit to ED or other physician for } \\
\text { worsening symptoms }\end{array}$ & 66 & Canada & ED & Initial ED visit & $26 \%(10)$ \\
\hline $\begin{array}{l}\text { Adams } \\
2000^{31}\end{array}$ & Return visit to ED & 362 visits & USA & ED & Initial ED visit & $22 \%(14)$ \\
\hline $\begin{array}{l}\text { Cydulka } \\
2003^{27}\end{array}$ & $\begin{array}{l}\text { Patient report of same or worsening } \\
\text { condition since last ED visit }\end{array}$ & 185 & $\begin{array}{l}\text { USA and } \\
\text { Canada, } \\
\text { MARC }\end{array}$ & ED & Initial ED visit & $14 \%(14)$ \\
\hline $\begin{array}{l}\text { Domenech } \\
2013^{32}\end{array}$ & $\begin{array}{l}\text { New exacerbation by pneumococci } \\
\text { with different serotype than index } \\
\text { exacerbation }\end{array}$ & $\begin{array}{l}\text { II } 6 \\
\text { exacerbations }\end{array}$ & Spain & Hospital & Not reported & $69.8 \%^{c}$ \\
\hline $\begin{array}{l}\text { Durmaz } \\
2015^{34}\end{array}$ & $\begin{array}{l}\text { Symptoms within } 5 \text { days of index } \\
\text { exacerbation }\end{array}$ & 196 & Turkey & ED & Initial ED visit & $27.6 \%(\mid 4)$ \\
\hline $\begin{array}{l}\text { Durmaz } \\
2015^{33}\end{array}$ & $\begin{array}{l}\text { Re-visit to ED for worsening } \\
\text { symptoms }\end{array}$ & 92 & Turkey & ED & Initial ED visit & $30 \%(14)$ \\
\hline $\begin{array}{l}\text { Hurst } \\
2009^{28}\end{array}$ & $\begin{array}{l}\text { Symptoms within } 5 \text { days of index } \\
\text { exacerbation }\end{array}$ & $\begin{array}{l}410 \\
\text { exacerbations }\end{array}$ & $\begin{array}{l}\text { UK, } \\
\text { London } \\
\text { COPD } \\
\text { Cohort }\end{array}$ & Outpatient & $\begin{array}{l}\text { Remission of } \\
\text { symptoms }\end{array}$ & $11 \%(5)$ \\
\hline $\begin{array}{l}\text { Kim } \\
2004^{35}\end{array}$ & Re-visit to ED or clinic & 140 & $\begin{array}{l}\text { USA and } \\
\text { Canada, } \\
\text { MARC }\end{array}$ & ED & Initial ED visit & $17 \%(14)$ \\
\hline $\begin{array}{l}\text { Minov } \\
2018^{36}\end{array}$ & $\begin{array}{l}\text { Worsening cough, expectoration, and } \\
\text { shortness of breath }\end{array}$ & 54 & Macedonia & Outpatient & $\begin{array}{l}\text { Remission of } \\
\text { symptoms }\end{array}$ & $7.4 \%(20)$ \\
\hline $\begin{array}{l}\text { Miravitlles } \\
200 I^{37}\end{array}$ & $\begin{array}{l}\text { Unscheduled visit leading to change in } \\
\text { drug prescription, an ED visit, or } \\
\text { hospital admission }\end{array}$ & 2414 & Spain & $\begin{array}{l}\text { Primary } \\
\text { care }\end{array}$ & $\begin{array}{l}\text { Initial primary care } \\
\text { visit }\end{array}$ & $21 \%(1 \mathrm{mo})$ \\
\hline $\begin{array}{l}\text { Miravitlles } \\
2003^{38}\end{array}$ & $\begin{array}{l}\text { Unscheduled office visit or } \\
\text { hospitalization for persistent or } \\
\text { worsening symptoms }\end{array}$ & 441 & Spain & $\begin{array}{l}\text { Outpatient } \\
\text { clinic }\end{array}$ & $\begin{array}{l}\text { Following initial } \\
\text { exacerbation } \\
\text { Initiation of } \\
\text { antibiotic therapy }\end{array}$ & $\begin{array}{l}34 \%(1 \mathrm{mo}) \\
\text { Mean time to } \\
\text { relapse: } 4.6(\mathrm{SD} 3.3)\end{array}$ \\
\hline $\begin{array}{l}\text { Stiell } \\
2018^{39}\end{array}$ & $\begin{array}{l}\text { Return to ED for any related } \\
\text { problem, with or without admission } \\
\text { to hospital }\end{array}$ & 1415 & Canada & $\begin{array}{l}\text { Hospital } \\
\text { and ED }\end{array}$ & $\begin{array}{l}\text { Unclear if hospital } \\
\text { discharge or } \\
\text { admission }\end{array}$ & $\begin{array}{l}\text { ED: } 21.8 \%(14 \mathrm{~d})^{\mathrm{d}} \\
\text { ED with admission: } \\
8.3 \%(14 \mathrm{~d})^{\mathrm{e}}\end{array}$ \\
\hline $\begin{array}{l}\text { Vondracek } \\
2006^{40}\end{array}$ & $\begin{array}{l}\text { Hospital admission, ED visit, or clinic } \\
\text { visit }\end{array}$ & 80 admissions & USA & Hospital & Discharge & $28 \%(30)$ \\
\hline
\end{tabular}

Notes: ${ }^{a} \mathrm{~N}$ indicates number of patients unless otherwise specified; ${ }^{\mathrm{b}}$ Days unless otherwise specified; ${ }^{\mathrm{C}}$ Not reported in Figure IA because no time horizon included; ${ }^{d}$ Defined as return to ED for any related problem; 'Defined as return to ED for any related problem followed by admission to hospital.

Abbreviations: COPD, chronic obstructive pulmonary disease; d, days; ED, emergency department; MARC, Medication Adherence Research in COPD Patients; mo, months; SD, standard deviation; UK, United Kingdom; USA, United States of America. 
Table 2 Characteristics of Studies Observing Recurrence or Re-Exacerbation Events Following an Exacerbation

\begin{tabular}{|c|c|c|c|c|c|c|}
\hline Author, Year & $\begin{array}{l}\text { Re-Exacerbation or } \\
\text { Recurrence Definition }\end{array}$ & $\mathbf{N}^{\mathbf{a}}$ & $\begin{array}{l}\text { Country, } \\
\text { Study }\end{array}$ & Setting & $\begin{array}{l}\text { Time Point From } \\
\text { Which } \\
\text { Re-Exacerbation } \\
\text { or Recurrence is } \\
\text { Measured }\end{array}$ & $\begin{array}{l}\text { Proportion with } \\
\text { Re-Exacerbation } \\
\text { or Recurrence } \\
\text { [Time Frame, } \\
(\text { Days) }]^{\text {b }}\end{array}$ \\
\hline $\begin{array}{l}\text { Bartziokas } \\
2014^{41}\end{array}$ & $\begin{array}{l}\text { The need for antibiotics and/or } \\
\text { OCS, visits to ED, and/or } \\
\text { hospitalizations }\end{array}$ & 314 & Greece & Hospital & Discharge $^{c}$ & $\begin{array}{l}\text { High uric acid } \\
50 \%(3 \mathrm{mo}), 80 \% \\
(6 \mathrm{mo}) \\
\text { Low uric acid } \\
15 \%(3 \mathrm{mo}), 60 \% \\
(6 \mathrm{mo})\end{array}$ \\
\hline $\begin{array}{l}\text { Bathoorn } \\
2017^{42}\end{array}$ & $\begin{array}{l}\text { Prescription of subsequent course } \\
\text { of corticosteroids within } 60 \text { days of } \\
\text { first course }\end{array}$ & $\begin{array}{l}1558 \\
\text { exacerbations }\end{array}$ & $\begin{array}{l}\text { The } \\
\text { Netherlands }\end{array}$ & $\begin{array}{l}\text { Primary } \\
\text { care }\end{array}$ & $\begin{array}{l}\text { First course of } \\
\text { prescribed } \\
\text { corticosteroids }^{d}\end{array}$ & $28 \%(60)$ \\
\hline Chang $2014^{43}$ & $\begin{array}{l}\text { Re-exacerbation after symptoms } \\
\text { from initial exacerbation returned } \\
\text { to pre-exacerbation level }\end{array}$ & 135 & China & Hospital & Discharge & $18.5 \%(56)$ \\
\hline Cushen $2016^{44}$ & $\begin{array}{l}\text { Requiring antibiotics and/or steroid } \\
\text { therapy }\end{array}$ & 62 & Ireland & Hospital & Discharge & $14.5 \%(14)$ \\
\hline Hu $2019^{45}$ & $\begin{array}{l}\text { Worsening respiratory symptoms } \\
\text { for } \geq 2 \text { consecutive days requiring } \\
\text { intervention or medication changes } \\
\text { after sustained relief from last } \\
\text { exacerbation of } \geq 14 \text { days }\end{array}$ & 686 & China & Hospital & Discharge & $15 \%(30)$ \\
\hline Hurst $2009^{28}$ & $\begin{array}{l}\text { New exacerbation after symptom } \\
\text { free for } 5 \text { days within } 8 \text { weeks of } \\
\text { previous exacerbation }\end{array}$ & $\begin{array}{l}410 \\
\text { exacerbations }\end{array}$ & $\begin{array}{l}\text { UK, London } \\
\text { COPD } \\
\text { Cohort }\end{array}$ & Outpatient & $\begin{array}{l}\text { Remission of } \\
\text { symptoms }^{\mathrm{e}}\end{array}$ & $\begin{array}{l}27.4 \%(56) \\
\text { Median days to re- } \\
\text { exacerbation or } \\
\text { recurrence: } 5 \text { [Q।, } \\
\text { Q3: } 3,8]\end{array}$ \\
\hline $\begin{array}{l}\text { Johannesdottir } \\
2013^{46}\end{array}$ & $\begin{array}{l}\text { Inpatient readmission or re-visit, } \\
\text { mechanical ventilation, or } \\
\text { simultaneous antibiotics and steroid } \\
\text { prescription }\end{array}$ & $\begin{array}{l}6240 \\
\text { exacerbations }\end{array}$ & Denmark & Hospital & Discharge & $\begin{array}{l}19.7 \%(30) \\
31.6 \%(60) \\
40.1 \%(90) \\
57.4 \%(180)\end{array}$ \\
\hline Liu $2015^{47}$ & $\begin{array}{l}\text { Sustained worsening of symptoms } \\
\text { for } \geq 2 \text { days requiring visit to doctor, } \\
E D \text {, and/or antibiotics, } \\
\text { corticosteroids, or both }\end{array}$ & 176 & China & Hospital & Discharge & $48.9 \%(90)$ \\
\hline Perera $2007^{48}$ & $\begin{array}{l}\text { New exacerbation after symptom } \\
\text { recovery from index exacerbation }\end{array}$ & 73 & $\begin{array}{l}\text { UK, London } \\
\text { COPD } \\
\text { Cohort }\end{array}$ & Outpatient & $\begin{array}{l}\text { Onset of index } \\
\text { exacerbation }^{f}\end{array}$ & $\begin{array}{l}22 \%(50) \\
\text { Median days to re- } \\
\text { exacerbation or } \\
\text { recurrence: } 9 \text { [Q I, } \\
\text { Q3: } 4,18]\end{array}$ \\
\hline Wang $2012^{49}$ & $\begin{array}{l}\text { Worsening cough, expectoration, } \\
\text { and shortness of breath }\end{array}$ & 136 & China & Hospital & Discharge & $\begin{array}{l}7.4 \%(30) \\
25 \%(90) \\
55.6 \%(180) \\
88.2 \%(365)\end{array}$ \\
\hline
\end{tabular}

(Continued) 
Table 2 (Continued).

\begin{tabular}{|l|l|l|l|l|l|l|}
\hline Author, Year & $\begin{array}{l}\text { Re-Exacerbation or } \\
\text { Recurrence Definition }\end{array}$ & $\mathbf{N}^{\mathbf{a}}$ & $\begin{array}{l}\text { Country, } \\
\text { Study }\end{array}$ & Setting & $\begin{array}{l}\text { Time Point From } \\
\text { Which } \\
\text { Re-Exacerbation } \\
\text { or Recurrence is } \\
\text { Measured }\end{array}$ & $\begin{array}{l}\text { Proportion with } \\
\text { Re-Exacerbation } \\
\text { or Recurrence } \\
{[\text { Time Frame, }} \\
\text { (Days) }]^{\mathbf{b}}\end{array}$ \\
\hline Yount 201950 & $\begin{array}{l}\text { Sustained worsening of COPD } \\
\text { symptoms beyond normal day-to- } \\
\text { day variations with acute onset } \\
\text { requiring change in regular } \\
\text { medication, admission for COPD, } \\
\text { and/or treatment with antibiotics or } \\
\text { corticosteroids }\end{array}$ & 85 & USA & $\begin{array}{l}\text { Hospital } \\
\text { inpatients } \\
\text { and } \\
\text { outpatients }\end{array}$ & $\begin{array}{l}\text { Enrollment into } \\
\text { the study }\end{array}$ & $17.6 \%(84)$ \\
\hline
\end{tabular}

Notes: a $\mathrm{N}$ represents patients unless otherwise noted; bays unless otherwise specified; 'Patients were evaluated on admission and at discharge by the study investigators and were followed up for I year; ${ }^{d}$ Unclear whether follow-up began at prescription start or end; ${ }^{\text {eAfter }} 5$ consecutive symptom-free days; ${ }^{\mathrm{f}} \mathrm{Not}$ explicitly mentioned in article, but for other outcomes such as non-recovery the starting point is onset of index exacerbation; ${ }^{\mathrm{g} M a x i m u m}$ of 3 days from start of treatment for patients recruited in outpatient setting and maximum of 6 days from treatment start for patients recruited in the inpatient setting.

Abbreviations: COPD, chronic obstructive pulmonary disease; ED, emergency department; mo, months; OCS, oral corticosteroids; QI, quartile I; Q3, quartile 3; UK, United Kingdom; USA, United States of America.

admission $^{60}$ to ninety days from hospital discharge. ${ }^{54}$ The proportion of patients with treatment failure ranged from $14.5 \%$ at/before seven days ${ }^{51}$ to $36.8 \%$ at/before 90 days. ${ }^{54}$ By 30 days (the most common timeframe), the proportion experiencing treatment failure ranged from $7.4 \%$ to $34.8 \%{ }^{55}$ - suggesting heterogeneity in the studies, potentially due to a range of definitions for treatment failure between studies.

\section{Non-Recovery and Time to Recovery}

The term non-recovery from an exacerbation was used as a definition for subsequent AECOPD events in 17 studies and was predominantly defined as either symptoms or peak expiratory flow not returning to baseline. ${ }^{10,27,28,36,38,48,57,63-72}$ Eight studies using the term non-recovery were set within the London COPD cohort, which used patient diary cards to assess recovery. Four of the studies were conducted in outpatient clinics, and two in the ED. One study did not report setting. Four studies, three in the London COPD cohort and one in GIANT, reported both time to recovery and proportion of patients non-recovered by a certain number of days post index. ${ }^{48,63,71,72}$

Timeframes for non-recovery definitions ranged from eight days from an unclear start point ${ }^{62}$ to three months from start of exacerbation. ${ }^{72}$ The proportion of patients who met the criteria for non-recovery ranged from $8.3 \%$ at 99 days $^{72}$ to $25 \%$ at 35 days. $^{71}$ Thirteen articles reported mean or median time to recovery rather than (or in addition to) the proportion of patients recovering. Most of these studies were based in the London COPD cohort and conducted in an outpatient setting. Median time to recovery of symptoms in the London COPD studies ranged from 7 days (Q1, Q3: 4, 14 days $^{71}$ to 10 days (Q1, Q3: 6, 18 days), ${ }^{72}$ with similar median time to recovery of peak expiratory flow of 5 and 6 days (Q1, Q3: 0, 14 days and 1, 14 days). ${ }^{71,72}$ One study reported mean time to "early" recovery ( $\leq 4$ days) and to "late" recovery ( $\geq 8$ days) of 3.1 days (SD: 0.9 days) and 102 days (SD: 2.5 days), respectively. ${ }^{63}$ Overall, median recovery time ranged from 5 days ${ }^{28}$ to 13 days ${ }^{30}$ and mean recovery time ranged from 3 days ${ }^{63}$ to 19 days. ${ }^{66}$

Six studies reported "time to recovery" only (ie, no proportions) in either an outpatient $(n=5)$ or cohort $(\mathrm{n}=1)$ study setting, with recovery defined as improvement in symptoms. Two studies measured time from the initial patient visit, three measured time from onset of exacerbation, and one measured time from the last COPD exacerbation. One study presented total recovery time and treated recovery time ${ }^{69}$ and one study presented time to recovery stratified by chronic bronchitis status. ${ }^{66}$ Mean time to recovery ranged from 6.3 days (SD 3.1 days) ${ }^{68}$ to 13.3 days (SD: 13.3 days) ${ }^{69}$ Median time to recovery ranged from 7 days (Q1, Q3: 0, 12 days $)^{67}$ to 13 days (Q1, Q3: 7, 29 days). ${ }^{73}$ 
Table 3 Characteristics of Studies Observing Treatment Failure Following an Exacerbation

\begin{tabular}{|c|c|c|c|c|c|c|}
\hline $\begin{array}{l}\text { Author, } \\
\text { Year }\end{array}$ & Treatment Failure Definition & $\mathbf{N}^{\mathrm{a}}$ & $\begin{array}{l}\text { Country, } \\
\text { Dataset }\end{array}$ & Setting & $\begin{array}{l}\text { Time Point } \\
\text { From Which } \\
\text { Treatment } \\
\text { Failure is } \\
\text { Measured }\end{array}$ & $\begin{array}{l}\text { Proportion with } \\
\text { Treatment } \\
\text { Failure [Time } \\
\text { Frame, } \\
\text { (Days)] }^{\text {b }}\end{array}$ \\
\hline $\begin{array}{l}\text { Beauchesne } \\
2008^{51}\end{array}$ & $\begin{array}{l}\text { Prolonged use of antibiotics, ED visit, or } \\
\text { hospitalization }\end{array}$ & $\begin{array}{l}\text { II } 80 \\
\text { exacerbations }\end{array}$ & Canada & $\begin{array}{l}\text { Home care } \\
\text { program }\end{array}$ & $\begin{array}{l}\text { Initiation of } \\
\text { antibiotic } \\
\text { treatment }\end{array}$ & $29.5 \%(30)$ \\
\hline $\begin{array}{l}\text { Crisafulli } \\
2016^{52}\end{array}$ & $\begin{array}{l}\text { Need for NIMV, ICU admission, new } \\
\text { course of antibiotics, or death }\end{array}$ & 110 & Spain & Hospital & Day 2 of admission & $14.5 \%(7)$ \\
\hline $\begin{array}{l}\text { Dewan } \\
2000^{53}\end{array}$ & $\begin{array}{l}\text { Readmission or re-visit requiring } \\
\text { a change of antibiotic }\end{array}$ & 107 & USA & Outpatient & $\begin{array}{l}\text { Commencement } \\
\text { of initial treatment }\end{array}$ & $26 \%(28)$ \\
\hline $\begin{array}{l}\text { Garcia-Sidro } \\
2015^{54}\end{array}$ & $\begin{array}{l}\text { Exacerbation with or without } \\
\text { readmission, or death }\end{array}$ & 106 & Spain & Hospital & Discharge & $36.8 \%(90)$ \\
\hline $\begin{array}{l}\text { Gaude } \\
2015^{55}\end{array}$ & $\begin{array}{l}\text { Return visit requiring change of } \\
\text { antibiotic or hospitalization }\end{array}$ & 115 & India & Hospital & Discharge & $34.8 \%(28)$ \\
\hline $\begin{array}{l}\text { Lindenauer } \\
2010^{56}\end{array}$ & $\begin{array}{l}\text { Mechanical ventilation after second } \\
\text { hospital day, death during } \\
\text { hospitalization, or readmission }\end{array}$ & 79,985 & $\begin{array}{l}\text { USA, } \\
\text { Premier } \\
\text { Perspective }\end{array}$ & Hospital & Discharge & $10.8 \%(30)$ \\
\hline $\begin{array}{l}\text { Miravitlles } \\
2005^{57}\end{array}$ & $\begin{array}{l}\text { Failure to return to baseline or need for } \\
\text { new treatment or medication }\end{array}$ & 1147 & Spain & $\begin{array}{l}\text { Primary } \\
\text { care }\end{array}$ & Initial visit & $15.1 \%(10)$ \\
\hline $\begin{array}{l}\text { Miravitlles } \\
2011^{58}\end{array}$ & $\begin{array}{l}\text { Absence of sign/symptom resolution, } \\
\text { worsening of signs/symptoms or death }\end{array}$ & 346 & Spain & Outpatients & Initial visit & $28.2 \%(30)$ \\
\hline $\begin{array}{l}\text { Miravitlles } \\
2013^{59}\end{array}$ & $\begin{array}{l}\text { Incomplete resolution, persistence, or } \\
\text { worsening of symptoms requiring new } \\
\text { course of antibiotics and/or OCS or } \\
\text { hospitalization }\end{array}$ & 260 & Spain & Outpatients & Initial visit & $\begin{array}{l}13.5 \%(28) \\
\text { Mean days to } \\
\text { treatment failure: } \\
7 \text { [SD 4.6] }\end{array}$ \\
\hline $\begin{array}{l}\text { Planquette } \\
2015^{60}\end{array}$ & $\begin{array}{l}\text { Increased mechanical ventilator support, } \\
\text { initiation of OCS, or death }\end{array}$ & III & France & $\begin{array}{l}\text { Hospital } \\
\text { (ICU) }\end{array}$ & Admission & $21.6 \%(2)$ \\
\hline $\begin{array}{l}\text { Rothberg } \\
2010^{61}\end{array}$ & $\begin{array}{l}\text { Initiation of hospital mechanical } \\
\text { ventilation, in-hospital death, or } \\
\text { readmission }\end{array}$ & 19,608 & $\begin{array}{l}\text { USA, } \\
\text { Premier } \\
\text { Perspective }\end{array}$ & Hospital & $\begin{array}{l}\text { Day } 2 \text { of } \\
\text { admission }^{c} \text {, or } \\
\text { discharge }\end{array}$ & $7.7 \%(30)$ \\
\hline $\begin{array}{l}\text { Rothberg } \\
2010^{62}\end{array}$ & $\begin{array}{l}\text { Initiation of hospital mechanical } \\
\text { ventilation, in-hospital death, or } \\
\text { readmission }\end{array}$ & 84,621 & $\begin{array}{l}\text { USA, } \\
\text { Premier } \\
\text { Perspective }\end{array}$ & Hospital & $\begin{array}{l}\text { Day } 2 \text { of } \\
\text { admission }{ }^{c} \text {, or } \\
\text { discharge }\end{array}$ & $10.2 \%(30)$ \\
\hline
\end{tabular}

Notes: ${ }^{a} \mathrm{~N}$ represents patients unless otherwise noted; ${ }^{\mathrm{b}}$ Days unless otherwise specified; ${ }^{\mathrm{c}}$ Starting point for treatment failure defined as initiation of mechanical ventilation at day 2 of hospital admission.

Abbreviations: ICU, intensive care unit; NIMV, non-invasive mechanical ventilation; OCS, oral corticosteroids; SD, standard deviation; USA, United States of America.

\section{Discussion}

In our systematic review, we found no single, distinct terms or definitions were used to define subsequent AECOPD events in the observational study literature, though most studies used one of four clustered terms and definitions: relapse, recurrence/re-exacerbation, treatment failure and non-recovery/time to recovery. Both heterogeneity and similarities were observed among terminologies of subsequent AECOPD, and in the time frames and the settings used to assess these subsequent AECOPD events. Despite some similarities, the heterogeneity we observed may hamper comparability and synthesis of these studies. 
Table 4 Characteristics of Studies Observing Non-Recovery or Time to Recovery Events Following an Exacerbation

\begin{tabular}{|c|c|c|c|c|c|c|}
\hline $\begin{array}{l}\text { Author, } \\
\text { Year }\end{array}$ & Non-Recovery or Recovery Definition & $\mathbf{N}^{\mathbf{a}}$ & $\begin{array}{l}\text { Countryl } \\
\text { Study }\end{array}$ & Setting & $\begin{array}{l}\text { Time Point } \\
\text { From Which } \\
\text { Non-Recovery } \\
\text { is Measured }\end{array}$ & 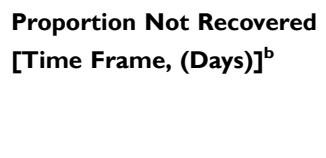 \\
\hline $\begin{array}{l}\text { Anzueto } \\
2012^{63}\end{array}$ & $\begin{array}{l}\text { Non-Recovery: Acute increase in I or more } \\
\text { symptoms (dyspnea, sputum volume, sputum } \\
\text { purulence) } \\
\text { Recovery: Clinician assessment that patient is } \\
\text { free of symptoms }\end{array}$ & 40,435 & GIANT $^{c}$ & $\begin{array}{l}\text { Not } \\
\text { reported }\end{array}$ & Unclear & $\begin{array}{l}15 \% \text { not recovered }(8) \\
\text { Mean time to recovery among } \\
\text { those with late and early } \\
\text { recovery: } 10.2 \text { [SD 2.5]; } 3.1 \\
\text { [SD 0.9 }^{\mathrm{d}}\end{array}$ \\
\hline $\begin{array}{l}\text { Cydulka } \\
2003^{27}\end{array}$ & $\begin{array}{l}\text { Non-Recovery: Symptoms at telephone } \\
\text { interview for } \geq 24 \text { hours or condition same or } \\
\text { worsened since last ED visit }\end{array}$ & 186 & MARC $^{e}$ & ED & Presenting to ED & $40 \%$ not recovered ( 14$)$ \\
\hline $\begin{array}{l}\text { Donaldson } \\
2003^{10}\end{array}$ & $\begin{array}{l}\text { Non-Recovery: PEF or symptom score below } \\
\text { baseline with symptoms recorded on diary card }\end{array}$ & $\begin{array}{l}\text { IIII } \\
\text { exacerbations }\end{array}$ & $\begin{array}{l}\text { UK, } \\
\text { London } \\
\text { COPD } \\
\text { Cohort }\end{array}$ & Outpatient & $\begin{array}{l}\text { Onset of index } \\
\text { exacerbation }\end{array}$ & $\begin{array}{l}\text { Symptom recovery: } \\
9.6 \% \text { not recovered (35) } \\
\text { PEF recovery: } \\
11.6 \% \text { not recovered (35) }\end{array}$ \\
\hline $\begin{array}{l}\text { Donaldson } \\
2015^{72}\end{array}$ & $\begin{array}{l}\text { Non-Recovery: Worsening symptoms or change } \\
\text { in medication required or PEF below baseline } \\
\text { Time to recovery: First of } 2 \text { consecutive } \\
\text { symptom-free days or PEF returned to baseline }\end{array}$ & $\begin{array}{l}3087 \\
\text { exacerbations }\end{array}$ & $\begin{array}{l}\text { UK, } \\
\text { London } \\
\text { COPD } \\
\text { Cohort }\end{array}$ & Outpatient & $\begin{array}{l}\text { Onset of index } \\
\text { exacerbation }\end{array}$ & $\begin{array}{l}\text { Symptom recovery: } \\
\text { 3.1\% not recovered (99 d) } \\
\text { Days to recovery: } \\
\text { Mean: } 14.7 \text { [SD: 14.2] } \\
\text { Median: I0 [QI, Q3: 6, I8] } \\
\text { PEF recovery: } \\
7.3 \% \text { not recovered (99) } \\
\text { Days to recovery: } \\
\text { Mean: } 10.3 \text { [SD I5] } \\
\text { Median: } 5 \text { [Q।, Q3: 0, I4] (0, } \\
\text { I4 d) }\end{array}$ \\
\hline $\begin{array}{l}\text { Perera } \\
2007^{48}\end{array}$ & $\begin{array}{l}\text { Non-Recovery: Total symptom score not } \\
\text { returned to baseline } \\
\text { Time to recovery: time from onset of } \\
\text { exacerbation to day on which a 3-day moving } \\
\text { average of symptom score returned to baseline }\end{array}$ & 73 & $\begin{array}{l}\text { UK, } \\
\text { London } \\
\text { COPD } \\
\text { Cohort }\end{array}$ & Outpatient & $\begin{array}{l}\text { Onset of index } \\
\text { exacerbation }\end{array}$ & $\begin{array}{l}23 \% \text { not recovered }(35) \\
\text { Median days to recovery: } 9 \\
{[Q \text { I, Q3: } 4,18]}\end{array}$ \\
\hline $\begin{array}{l}\text { Seemungal } \\
2000^{71}\end{array}$ & $\begin{array}{l}\text { Non-Recovery: PEF or symptoms not returning } \\
\text { to baseline } \\
\text { Time to recovery: symptoms or PEF returned } \\
\text { to baseline }\end{array}$ & $\begin{array}{l}504 \\
\text { exacerbations }\end{array}$ & $\begin{array}{l}\text { UK, } \\
\text { London } \\
\text { COPD } \\
\text { Cohort }\end{array}$ & Outpatient & $\begin{array}{l}\text { Onset of index } \\
\text { exacerbation }\end{array}$ & $\begin{array}{l}\text { Symptom recovery: } \\
\text { 13.9\% (35) } \\
9.1 \%(91) \\
\text { Median days to recovery: } 7 \text { (QI, } \\
\text { Q3: 4, I4) } \\
\text { PEF recovery: } \\
24.8 \%(35) \\
19.8 \%(91) \\
\text { Median days to recovery: } 6 \text { (QI, } \\
\text { Q3; I, } 14 \text { ) }\end{array}$ \\
\hline Tsai $2009^{70}$ & $\begin{array}{l}\text { Non-Recovery: Patient report of COPD status } \\
\text { as much worse, a little worse, or about the } \\
\text { same during telephone interview }\end{array}$ & 330 & MARC & ED & Presenting to ED & $18 \%(14)$ \\
\hline
\end{tabular}

(Continued) 
Table 4 (Continued).

\begin{tabular}{|c|c|c|c|c|c|c|}
\hline $\begin{array}{l}\text { Author, } \\
\text { Year }\end{array}$ & Non-Recovery or Recovery Definition & $\mathbf{N}^{\mathbf{a}}$ & $\begin{array}{l}\text { Countryl } \\
\text { Study }\end{array}$ & Setting & $\begin{array}{l}\text { Time Point } \\
\text { From Which } \\
\text { Non-Recovery } \\
\text { is Measured }\end{array}$ & $\begin{array}{l}\text { Proportion Not Recovered } \\
{[\text { Time Frame, (Days) }]^{\mathrm{b}}}\end{array}$ \\
\hline $\begin{array}{l}\text { Aaron } \\
2012^{73}\end{array}$ & $\begin{array}{l}\text { Time to Recovery: first date of } 5 \text { consecutive } \\
\text { days where self-reported symptoms return to } \\
\text { usual baseline }\end{array}$ & $\begin{array}{l}1995 \\
\text { exacerbations }\end{array}$ & $\begin{array}{l}\text { UK, } \\
\text { London } \\
\text { COPD } \\
\text { Cohort }\end{array}$ & Outpatient & $\begin{array}{l}\text { Onset of index } \\
\text { exacerbation }\end{array}$ & $\begin{array}{l}\text { Sudden onset of index } \\
\text { exacerbation: } \\
\text { Median days to recovery: II } \\
\text { [QI, Q3: 6, 22] } \\
\text { Gradual onset of index } \\
\text { exacerbation: } \\
\text { Median days to recovery: } 13 \\
{[Q 1, \text { Q3: 7, 29] }}\end{array}$ \\
\hline $\begin{array}{l}\text { Farias } \\
2019^{69}\end{array}$ & $\begin{array}{l}\text { Treated recovery time: time elapsed between } \\
\text { medication start date and exacerbation end date } \\
\text { Exacerbation recovery time: time elapsed } \\
\text { between exacerbation start and end date }\end{array}$ & $\begin{array}{l}68 \\
\text { exacerbations }\end{array}$ & Canada & Outpatient & Initial study visit & $\begin{array}{l}\text { Mean days of treated recovery } \\
\text { time: } 10.4 \text { [SD 10.5] } \\
\text { Mean days of exacerbation } \\
\text { recovery time: } 13.3 \text { [SD 13.3] }\end{array}$ \\
\hline $\begin{array}{l}\text { Hurst } \\
2009^{28}\end{array}$ & $\begin{array}{l}\text { Time to Recovery: number of days from } \\
\text { exacerbation onset to first of } 2 \text { consecutive } \\
\text { symptom-free days }\end{array}$ & $\begin{array}{l}410 \\
\text { exacerbations }\end{array}$ & $\begin{array}{l}\text { UK, } \\
\text { London } \\
\text { COPD } \\
\text { Cohort }\end{array}$ & Outpatient & $\begin{array}{l}\text { Onset of index } \\
\text { exacerbation }\end{array}$ & $\begin{array}{l}\text { Median time to recovery: } 5 \\
\text { [QI, Q3: 3, 8] }\end{array}$ \\
\hline $\begin{array}{l}\text { Liang } \\
2017^{66}\end{array}$ & $\begin{array}{l}\text { Time to Recovery: self-reported recovery } \\
\text { period }\end{array}$ & 890 & China & $\begin{array}{l}\text { Cohort } \\
\text { study }\end{array}$ & $\begin{array}{l}\text { Following last } \\
\text { COPD } \\
\text { exacerbation }\end{array}$ & $\begin{array}{l}\text { Mean days to recovery among } \\
\text { patients with bronchitis: } 19 \\
\text { [SD: } 16.2] \\
\text { Mean days to recovery among } \\
\text { patients without bronchitis: } \\
\text { I5.2 [SD } 14.7]\end{array}$ \\
\hline $\begin{array}{l}\text { Mackay } \\
2014^{67}\end{array}$ & $\begin{array}{l}\text { Time to Recovery: number of days that major } \\
\text { lower airway symptoms (dyspnea, sputum } \\
\text { volume, sputum purulence) were still being } \\
\text { recorded }\end{array}$ & $\begin{array}{l}128 \\
\text { exacerbations }\end{array}$ & $\begin{array}{l}\text { UK, } \\
\text { London } \\
\text { COPD } \\
\text { Cohort }\end{array}$ & Outpatient & $\begin{array}{l}\text { Onset of } \\
\text { exacerbation }\end{array}$ & $\begin{array}{l}\text { Median days to recovery: } 7 \\
\text { [QI, Q3: } 0,12]\end{array}$ \\
\hline $\begin{array}{l}\text { Minov } \\
2018^{36}\end{array}$ & $\begin{array}{l}\text { Time to Recovery: Resolution of cardinal } \\
\text { symptoms or return to baseline severity }\end{array}$ & 54 & Macedonia & Outpatient & $\begin{array}{l}\text { Onset of index } \\
\text { exacerbation }\end{array}$ & $\begin{array}{l}\text { Mean days to recovery: } 5.2 \\
{[S D \quad 1.1]}\end{array}$ \\
\hline $\begin{array}{l}\text { Miravitlles } \\
2003^{38}\end{array}$ & $\begin{array}{l}\text { Time to Recovery: number of days required for } \\
\text { symptoms to return to baseline }\end{array}$ & 441 & Spain & $\begin{array}{l}\text { Outpatient } \\
\text { clinic }\end{array}$ & $\begin{array}{l}\text { Following initial } \\
\text { exacerbation } \\
\text { Initiation of } \\
\text { antibiotic therapy }\end{array}$ & $\begin{array}{l}\text { Mean days to recovery: } 4.6 \\
\text { [SD 3.3] }\end{array}$ \\
\hline $\begin{array}{l}\text { Miravitlles } \\
2005^{57}\end{array}$ & $\begin{array}{l}\text { Time to Recovery: number of days for } \\
\text { symptoms to return to baseline }\end{array}$ & 1147 & Spain & $\begin{array}{l}\text { Primary } \\
\text { care }\end{array}$ & $\begin{array}{l}\text { Onset of index } \\
\text { exacerbation }\end{array}$ & Median: 5 days $^{g}$ \\
\hline $\begin{array}{l}\text { Miravitlles } \\
2009^{68}\end{array}$ & $\begin{array}{l}\text { Time to Recovery: physician assessment of } \\
\text { antibiotic therapy; patient reports number of } \\
\text { days to feeling better }\end{array}$ & 9225 & GIANT $^{\mathrm{h}}$ & Outpatient & Initial clinic visit & $\begin{array}{l}\text { Physician assessed: } 6.3 \text { [SD: } 3.1] \\
\text { Patient report: } 7.3 \text { [SD 3.1] }\end{array}$ \\
\hline $\begin{array}{l}\text { Wilkinson } \\
2004^{65}\end{array}$ & $\begin{array}{l}\text { Time to Recovery: time for } 3 \text {-day moving } \\
\text { average of total daily symptom count to return } \\
\text { to baseline }\end{array}$ & $\begin{array}{l}1099 \\
\text { exacerbations }\end{array}$ & $\begin{array}{l}\text { UK, } \\
\text { London } \\
\text { COPD } \\
\text { Cohort }\end{array}$ & Outpatient & $\begin{array}{l}\text { Onset of } \\
\text { exacerbation }\end{array}$ & $\begin{array}{l}\text { Median days to recovery: } 10.7 \\
\text { [QI, Q3: 7, 14] }\end{array}$ \\
\hline
\end{tabular}

Notes: ${ }^{a} \mathrm{~N}$ represents patients unless otherwise noted; ${ }^{\mathrm{b} D a y s}$ unless otherwise specified; ' $\mathrm{G} I \mathrm{ANT}$ study, worldwide; ${ }^{\mathrm{d}}$ Late recovery defined as $\geq 8$ days, early recovery defined as $\leq 4$ days; "MARC study, USA and Canada; 'Sudden exacerbations had an onset of 0 days and "gradual" exacerbations had an onset of 4 days with starting point after 5 consecutive days free of symptoms; ${ }^{\mathrm{g}} \mathrm{No}$ measure of spread reported; ${ }^{\mathrm{h}} \mathrm{GIANT}$ study, European.

Abbreviations: COPD, chronic obstructive pulmonary disease; ED, emergency department; MARC, Medication Adherence Research in COPD Patients; PEF, peak expiratory flow; QI, quartile I; Q3, quartile 3; SD, standard deviation; UK, United Kingdom. 
Table 5 Summary of the Four Terms Identified to Describe Subsequent AECOPD Events

\begin{tabular}{|l|l|}
\hline Relapse & $\begin{array}{l}\text { Typically defined as a re-visit to the } \\
\text { emergency department or other physician } \\
\text { visit for worsening symptoms within 5-30 } \\
\text { days of presentation to clinician }\end{array}$ \\
\hline $\begin{array}{l}\text { Re-exacerbation } \\
\text { /recurrence }\end{array}$ & $\begin{array}{l}\text { Subsequent exacerbation typically within I-6 } \\
\text { months from hospital discharge or following } \\
\text { X consecutive days free of recorded } \\
\text { symptoms }\end{array}$ \\
\hline Treatment failure & $\begin{array}{l}\text { Definition typically included death and/or was } \\
\text { related to in-hospital failure. Often } \\
\text { overlapping with relapse and non-recovery } \\
\text { terms/definitions. Predominantly measured in } \\
\text { hospital settings within I month of either } \\
\text { initial AECOPD visit or discharge }\end{array}$ \\
\hline $\begin{array}{l}\text { Non-recovery/time } \\
\text { to recovery }\end{array}$ & $\begin{array}{l}\text { (Includes late recovery, non-recovery and } \\
\text { ongoing AECOPD.) Non-recovery typically } \\
\text { defined using daily diaries as not returning to } \\
\text { baseline within 30-90 days from onset of initial } \\
\text { AECOPD. Time to recovery often defined } \\
\text { based on self-reported symptom recording, and } \\
\text { often specified a return to baseline }\end{array}$ \\
\hline
\end{tabular}

Abbreviation: AECOPD, acute exacerbation of chronic obstructive pulmonary disease.

In the literature, the distinction between the terms recurrence and re-exacerbation was unclear, and so these two terms were grouped in our analysis. Definitions for treatment failure and relapse often overlapped, such as a return to the ED or a hospital readmission. Definitions for non-recovery were generally distinct, with half of the studies using the term "non-recovery" set in the same cohort. Only one study elucidated the importance of the difference between the definitions of relapse and recurrence, where relapse is treatment failure of a first exacerbation and recurrence is a subsequent AECOPD after successful treatment. ${ }^{28}$

Terms used to define subsequent AECOPD-related events seem to be chosen pragmatically rather than based on any existing standards, and may be driven by external considerations (eg, incentives/penalties associated with readmissions for COPD within 30 days such as the Hospital Readmission Reduction Program in the US ${ }^{74}$ rather than event time course or disease phenotypes. These factors may contribute to the observed heterogeneity, making definitions of subsequent AECOPDs dependent on healthcare setting and partly explaining the large variation in estimates of subsequent AECOPD-related events defined using the four terminology and definition clusters. The available evidence suggests that the use of event- or symptom-based approaches may lead to substantially different conclusions regarding occurrence of exacerbations. ${ }^{75}$

In general, the time frames for observing an AECOPD described as a relapse were shorter (up to 30 days) than those for recurrences/re-exacerbations (up to 365 days); time frames for treatment failure and non-recovery were similar (up to approximately 90 days). For all four "subsequent AECOPD" terminology groups, the defined "end" of an initial exacerbation (ie, starting point for observing subsequent events) was variable but frequently included start or end of hospital admission or an ED visit. Reexacerbation/recurrence was commonly used in the studies with follow-up after hospital admission. The term "treatment failure" was also used frequently in the hospital setting. Non-recovery and relapse study settings were heterogenous.

We observed that the choice of the starting point for measuring subsequent AECOPD can have an important impact on estimates of the proportion of patients experiencing these subsequent events. For example, as a patient cannot experience a re-exacerbation whilst their index exacerbation is ongoing, the proportion of patients experiencing a relapse within 30 days of an index exacerbation will be higher when the 30-day timeframe begins after the exacerbation has ended than when the timeframe begins 5 days after the start of steroid treatment, for example. Exacerbation duration is variable and can be lengthy in some patients and may depend on severity, therefore estimates of subsequent AECOPD will be biased when looking for subsequent events before a patient has recovered or returned to baseline from their initial exacerbation. ${ }^{22}$

Similarly, exacerbation recovery will appear longer when measured from the onset of symptoms than upon hospital admission. Most of the studies using the term nonrecovery used onset of exacerbation as a starting point; however, all these studies were set in the London COPD cohort. The other two studies using non-recovery were also in the same cohort (Medication Adherence Research in COPD Patients cohort) and used presentation to the ED as the starting point. The starting point for re-exacerbation /recurrence in the hospital setting was primarily hospital discharge. Of note, one study set in the London COPD cohort used the term re-exacerbation/recurrence with a starting point as five days free of all recorded symptoms ${ }^{28}$ while another study also using re- 


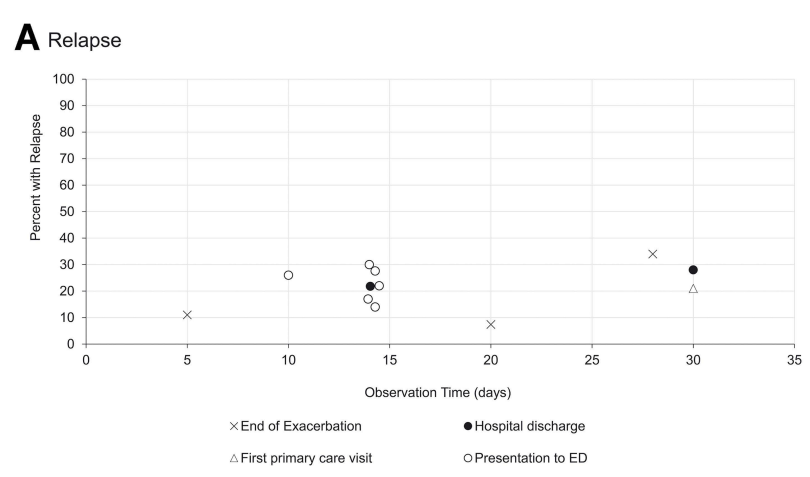

B Recurrence / re-exacerbation

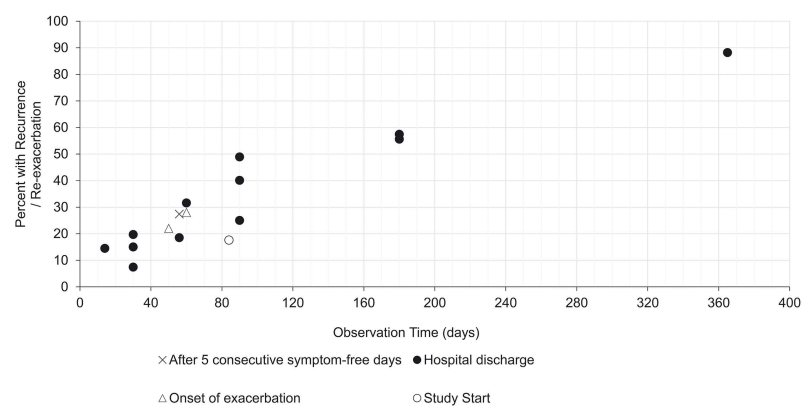

C Treatment Failure

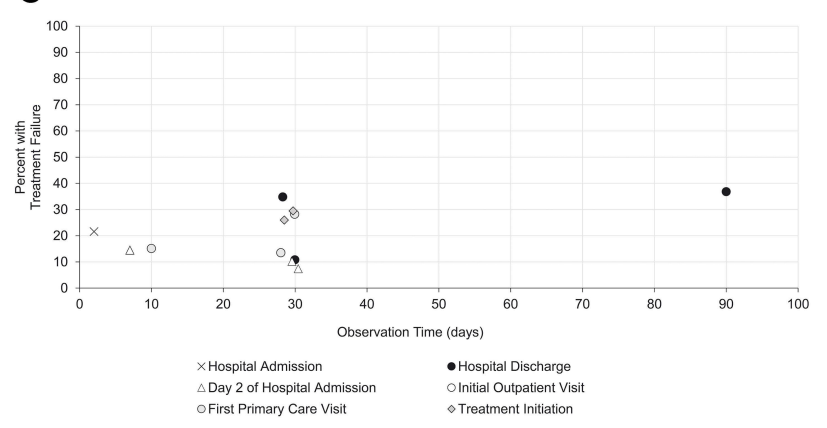

D Non-recovery

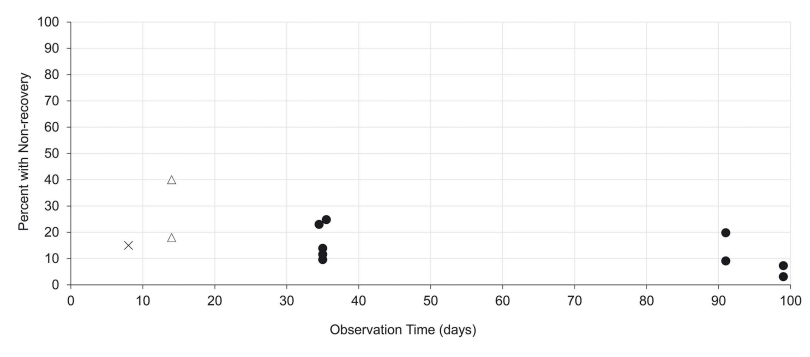

XNot Reported $\quad$ Onset of Exacerbation $\triangle$ Presentation to ED

Figure 2 Reported proportions of patients with (A) relapse event, (B) recurrence/re-exacerbation event, (C) treatment/clinical failure, and (D) non-recovery, by starting point of observation time frame. (A) Includes estimates from 12 observational studies reporting outcomes using a relapse term and definition. Defined as a re-visit to the emergency department or physician for a worsening of symptoms but typically not differentiated from "recurrence" or "re-exacerbation". One study used two definitions for relapse at 14 days and thus has two data points. Domenach (2013) is not plotted because the study did not report a timeframe. (B) Includes estimates from I0 observational studies reporting outcomes using a recurrence/re-exacerbation term and definition. Mostly defined as a "re-exacerbation" $(n=3)$, a prescription course of corticosteroids or antibiotics $(n=3)$, or as a composite outcome $(n=3)$ including death or AECOPD treatment and/or remission. Some studies $(n=4)$ required recovery of initial AECOPD prior to subsequent AECOPD. Two studies have data points at several time horizons (30,90, 180, and 365 days from Wang et al [2012] and 30,60, 90, and I80 days from Johannesdottir et al [2013]). Bartziokas et al (2014) reported proportion at 3 months and 6 months in "low uric acid" and "high uric acid" groups, but not overall, and therefore these data points are not presented in the figure. (C) Includes estimates from 12 observational studies reporting outcomes using a treatment failure term and definition with some definitions similar to relapse and non-recovery. Often included death $(n=7)$ and/or was related to in-hospital treatment failure $(n=6)$. (D) Includes estimates from seven observational studies reporting outcomes using a non-recovery term and definition. Some studies $(n=2)$ reported estimates at multiple time points and using different methods of measurement (eg, non-recovery ascertained daily symptom score reports or spirometry). Most were from the London COPD cohort. Two studies have multiple data points due to different measurement methods (symptom report and peak expiratory flow, Donaldson [2015] and Seemungal [2000]) over several time horizons (Seemungal [2000] at 35 days and 91 days).

Abbreviations: AECOPD, acute exacerbation of chronic obstructive pulmonary disease; ED, emergency department. 
exacerbation/recurrence and set in the London CODP cohort used onset of exacerbation, similar to the London COPD studies using non-recovery. ${ }^{48}$

In addition to heterogeneity in the terms, timeframes and starting points for subsequent AECOPD events, we also observed heterogeneity in the study setting. Studies using the term relapse were often set in the ED, whilst most studies conducted in the hospital setting used the term treatment failure. Overall, this heterogeneity has driven variation in the proportion of patients experiencing subsequent events. For example, the proportion of patients with exacerbation relapse ranged from $7.4 \%$ at 20 days $^{36}$ to $34 \%$ at one month, ${ }^{38}$ with variability (due to differing starting point for follow-up) appearing to have a greater impact on the relapse estimate then the length of follow-up (intuitively, we would expect to see greater proportions of patients experiencing relapse when the length of follow-up for relapse is increased).

There are several potential explanations for our finding that no standard definitions exist. First, exacerbations are heterogenous in terms of symptoms, etiology (bacterial vs viral, specific pathogens vs common pollutants), and time course. ${ }^{71,76,77}$ Second, there is variation in data sources, primary versus secondary data collection, and availability of specific data to define a re-exacerbation (eg, prescriptions for oral corticosteroids, self-reported symptoms). Finally, specific research questions often drive the type of data collected, and variability by definitions used in different guidelines and countries can contribute to heterogeneity.

Change begins with a recognition of the need for change, and this paper demonstrates that need by highlighting the large amount of heterogeneity between studies. Our finding of common core domains for each definition may be useful for developing an agreed consensus definition for classifying recurring exacerbations, treatment failure, and recovery from AECOPD. As a first step, we believe clear exacerbation definitions are required ${ }^{19,24}$ and that these should be determined by a consensus of experts (for example through a Delphi study) and cascaded via global guideline groups. Then, specific definitions are needed to enable differentiation between distinct AECOPD events. Without standard definitions, our ability to advance the understanding and natural history of AECOPD is impaired.

Strengths of this study include the systematic approach, and the comprehensive, international scope of the literature review. Additionally, studies were not excluded on the basis of quality which allowed us to describe the breadth of definitions currently in the literature. Nonetheless, there are limitations of our research which should be considered when interpreting our findings. First, studies where subsequent AECOPDs were defined as hospital readmission alone were excluded. Second, the included studies were not always explicit in definitions, particularly regarding the index date for recurrent AECOPD events which makes interpretation of the proportion of patients experiencing a subsequent AECOPD difficult. Third, subsequent AECOPD definitions may also differ to randomized clinical trials. This review focused on observational studies; however, a review of the terminology used in randomized clinical trials was presented previously and may better inform how terminology can be standardized to allow for better comparison of treatments. ${ }^{78}$

\section{Conclusion}

In conclusion, our systematic review demonstrates that the concept of subsequent AECOPD is ill-defined in the observational study literature, thus emphasizing the need for rigorous attempts to reach a consensus on a more precise and objective definition for subsequent AECOPDs. Use of standardized terminology and definitions may aid comparability and synthesis of studies, thus improving the understanding of the natural history of AECOPD.

\section{Acknowledgments}

We would like to thank Merike Verrijp, Jennifer Euewijk, and Judith van den Bosch (Pallas Health Research and Consulting, The Netherlands) for their part in conducting early literature searches and identifying articles meeting the inclusion criteria. We would also like to acknowledge Maggie Tabberer for her contribution to the project.

\section{Author Contributions}

VSB and HM contributed to the conception and design of the study, data acquisition, data analysis and interpretation. BMD contributed to the data acquisition, analysis and interpretation. WHM, BMD and WF contributed to the data analysis and interpretation. All authors made critical revisions to the draft versions of manuscript and approved the final manuscript, reviewed and agreed on all versions of the article before submission, during revisions, the final version accepted for publication, and any significant changes introduced at the proofing stage. All authors agreed on the journal to which the article would be 
submitted and agree to take responsibility and be accountable for the contents of the article.

\section{Funding}

This study was funded by GlaxoSmithKline (study number LS2859).

\section{Disclosure}

WAF and VSB are employees of, and hold shares in, GlaxoSmithKline plc. WHM and HM were employees of GlaxoSmithKline plc. at the time the study was conducted; WHM and HM are currently employees of AstraZeneca. $\mathrm{BMD}$ is a PhD candidate at University of North Carolina at Chapel Hill and works for GlaxoSmithKline plc. as a Research Assistant. The authors report no other conflicts of interest in this work.

\section{References}

1. World Health Organization. Global health estimates 2019: the top 10 causes of death, 2000-2019; 2020. Available from: https://www.who. int/data/global-health-estimates. Accessed November 24, 2021.

2. Global Burden of Disease Project Collaborators. Global, regional, and national age-sex specific mortality for 264 causes of death, 1980-2016: a systematic analysis for the Global Burden of Disease Study 2016. Lancet. 2017;390(10100):1151-1210. doi:10.1016/ S0140-6736(17)32152-9

3. Wedzichia JA, Brill SE, Allinson JP, Donaldson GC. Mechanisms and impact of the frequent exacerbator phenotype in chronic obstructive pulmonary disease. BMC Med. 2013;11:18. doi:10.1186/17417015-11-181

4. Mathioudakis AG, Janssens W, Sivapalan P, et al. Acute exacerbations of chronic obstructive pulmonary disease: in search of diagnostic biomarkers and treatable traits. Thorax. 2020;75(6):520-527. doi:10.1136/thoraxjnl-2019-214484

5. Wedzicha JA, Seemungal TA. COPD exacerbations: defining their cause and prevention. Lancet (London, England). 2007;370 (9589):786-796. doi:10.1016/S0140-6736(07)61382-8

6. National Institute for Health and Care Excellence. Chronic obstructive pulmonary disease in over 16s: diagnosis and management; 2018. Available from: https://www.nice.org.uk/guidance/ng115/chap ter/Recommendations. Accessed November 24, 2021.

7. Rothnie KJ, Müllerová H, Smeeth L, Quint JK. Natural history of chronic obstructive pulmonary disease exacerbations in a general practice-based population with chronic obstructive pulmonary disease. Am J Respir Crit Care Med. 2018;198(4):464-471.

8. Hurst JR, Vestbo J, Anzueto A, et al. Susceptibility to exacerbation in chronic obstructive pulmonary disease. $N$ Engl $J$ Med. 2010;363:1128-1138. doi:10.1056/NEJMoa0909883

9. Soler-Cataluña JJ, Martínez-García MA, Román Sánchez P, Salcedo E, Navarro M, Ochando R. Severe acute exacerbations and mortality in patients with chronic obstructive pulmonary disease. Thorax. 2005;60(11):925-931. doi:10.1136/ thx.2005.040527

10. Donaldson GC, Seemungal TA, Patel IS, Lloyd-Owen SJ, Wilkinson TM, Wedzicha JA. Longitudinal changes in the nature, severity and frequency of COPD exacerbations. Eur Respir J. 2003;22(6):931-936. doi:10.1183/09031936.03.00038303
11. Dransfield MT, Kunisaki KM, Strand MJ, et al. Acute exacerbations and lung function loss in smokers with and without chronic obstructive pulmonary disease. Am J Respir Crit Care Med. 2017;195 (3):324-330. doi:10.1164/rccm.201605-1014OC

12. Whittaker HR, Pimenta JM, Jarvis D, Kiddle SJ, Quint JK. Characteristics associated with accelerated lung function decline in a primary care population with chronic obstructive pulmonary disease. Int J Chron Obstruct Pulmon Dis. 2020;15:3079-3091. doi:10.2147/COPD.S278981

13. Seemungal TA, Donaldson GC, Paul EA, Bestall JC, Jeffries DJ, Wedzicha JA. Effect of exacerbation on quality of life in patients with chronic obstructive pulmonary disease. Am J Respir Crit Care Med. 1998;157(5 Pt 1):1418-1422. doi:10.1164/ ajrccm.157.5.9709032

14. Halpin DM, Miravitlles M, Metzdorf N, Celli B. Impact and prevention of severe exacerbations of COPD: a review of the evidence. Int J Chron Obstruct Pulmon Dis. 2017;12:2891-2908. doi:10.2147/ COPD.S139470

15. Ramsey SD, Sullivan SD. The burden of illness and economic evaluation for COPD. Eur Respir J Suppl. 2003;21:29s-35s. doi:10.1183/09031936.03.00078203

16. Strassels SA, Smith DH, Sullivan SD, Mahajan PS. The costs of treating COPD in the United States. Chest. 2001;119(2):344-352. doi:10.1378/chest.119.2.344

17. Wedzicha C, Miravitlles M, Hurst JR, et al. Management of COPD exacerbations: a European Respiratory Society/American Thoracic Society guideline. Eur Respir J. 2017;49(3):1600791. doi:10.1183/ 13993003.00791-2016

18. Anthonisen NR, Manfreda J, Warren CP, Hershfield ES, Harding GK, Nelson NA. Antibiotic therapy in exacerbations of chronic obstructive pulmonary disease. Ann Intern Med. 1987;106(2):196-204. doi:10.7326/0003-4819-106-2-196

19. Montes de Oca M, Laucho-Contreras ME. Is it time to change the definition of acute exacerbation of chronic obstructive pulmonary disease? What do we need to add? Med Sci. 2018;6(2):50. doi:10.3390/medsci6020050

20. Leidy NK, Sexton CC, Jones PW, et al. Measuring respiratory symptoms in clinical trials of COPD: reliability and validity of a daily diary. Thorax. 2014;69(5):443-449. doi:10.1136/thoraxjnl-2013-204428

21. O'Reilly JF, Williams AE, Holt K, Rice L. Defining COPD exacerbations: impact on estimation of incidence and burden in primary care. Prim Care Respir J. 2006;15(6):346-353. doi:10.1016/j. pcrj.2006.08.009

22. Murray LT, Leidy NK. The short-term impact of symptom-defined COPD exacerbation recovery on health status and lung function. Chron Obstruct Pulmon Dis. 2018;5(1):27-37. doi:10.15326/ jcopdf.5.1.2017.0166

23. Rothnie KJ, Müllerová H, Hurst JR, et al. Validation of the recording of acute exacerbations of COPD in UK primary care electronic healthcare records. PLoS One. 2016;11(3):e0151357. doi:10.1371/ journal.pone. 0151357

24. Pauwels R, Calverley P, Buist AS, et al. COPD exacerbations: the importance of a standard definition. Respir Med. 2004;98(2):99-107. doi:10.1016/j.rmed.2003.09.001

25. Caramori G, Adcock IM, Papi A. Clinical definition of COPD exacerbations and classification of their severity. South Med J. 2009;102(3):277-282. doi:10.1097/SMJ.0b013e3181836b73

26. Page MJ, McKenzie JE, Bossuyt PM, et al. The PRISMA 2020 statement: an updated guideline for reporting systematic reviews. BMJ. 2021;372:n71. doi:10.1136/bmj.n71

27. Cydulka RK, Rowe BH, Clark S, Emerman CL, Camargo CA Jr. Emergency department management of acute exacerbations of chronic obstructive pulmonary disease in the elderly: the Multicenter Airway Research Collaboration. J Am Geriatr Soc. 2003;51(7):908-916. doi:10.1046/j.1365-2389.2003.51302.x 
28. Hurst JR, Donaldson GC, Quint JK, Goldring JJ, Baghai-Ravary R, Wedzicha JA. Temporal clustering of exacerbations in chronic obstructive pulmonary disease. Am $J$ Respir Crit Care Med. 2009;179(5):369-374. doi:10.1164/rccm.200807-1067OC

29. Matkovic Z, Huerta A, Soler N, et al. Predictors of adverse outcome in patients hospitalised for exacerbation of chronic obstructive pulmonary disease. Respir Int Rev Thorac Dis. 2012;84(1):17-26. doi:10.1159/000335467

30. Aaron SD, Vandemheen KL, Clinch JJ, et al. Measurement of short-term changes in dyspnea and disease-specific quality of life following an acute COPD exacerbation. Chest. 2002;121 (3):688-696. doi:10.1378/chest.121.3.688

31. Adams SG, Melo J, Luther M, Anzueto A. Antibiotics are associated with lower relapse rates in outpatients with acute exacerbations of COPD. Chest. 2000;117(5):1345-1352. doi:10.1378/chest.117.5.1345

32. Domenech A, Ardanuy C, Pallares R, et al. Some pneumococcal serotypes are more frequently associated with relapses of acute exacerbations in COPD patients. PLoS One. 2013;8(3):e59027. doi:10.1371/journal.pone.0059027

33. Durmaz D, Goksu E, Kilic T, Ozbudak O, Eray O. The role of nitric oxide in predicting revisit of patients with exacerbated chronic obstructive pulmonary disease. J Emerg Med. 2015;48(2):247-253. doi:10.1016/j.jemermed.2014.06.026

34. Durmaz D, Goksu E, Yildiz G, et al. The factors influencing relapse in patients presenting to the emergency department with COPD exacerbation. Turk J Emerg Med. 2015;15(2):59-63. doi:10.5505/ 1304.7361.2014.37791

35. Kim S, Emerman CL, Cydulka RK, Rowe BH, Clark S, Camargo CA. Prospective multicenter study of relapse following emergency department treatment of COPD exacerbation. Chest. 2004;125(2):473-481. doi:10.1378/chest.125.2.473

36. Minov J, Stoleski S, Petrova T, Vasilevska K, Mijakoski D, Bislimovska-Karadzhinska J. Moxifloxacin in the outpatient treatment of moderate exacerbations of chronic obstructive pulmonary disease. Open Access Macedon J Med Sci. 2018;6(11):2017-2022. doi:10.3889/oamjms.2018.445

37. Miravitlles M, Murio C, Guerrero T. Factors associated with relapse after ambulatory treatment of acute exacerbations of chronic bronchitis. DAFNE Study Group. Eur Respir J. 2001;17(5):928-933. doi:10.1183/09031936.01.17509280

38. Miravitlles M, Zalacain R, Murio C, et al. Speed of recovery from acute exacerbations of chronic obstructive pulmonary disease after treatment with antimicrobials: results of a two-year study. Clin Drug Investig. 2003;23(7):439-450. doi:10.2165/00044011-20032307000003

39. Stiell IG, Perry JJ, Clement CM, et al. Clinical validation of a risk scale for serious outcomes among patients with chronic obstructive pulmonary disease managed in the emergency department. CMAJ. 2018;190(48):E1406-E1413. doi:10.1503/cmaj.180232

40. Vondracek SF, Hemstreet BA. Retrospective evaluation of systemic corticosteroids for the management of acute exacerbations of chronic obstructive pulmonary disease. Am J Health Sys Pharm. 2006;63 (7):645-652. doi:10.2146/ajhp050316

41. Bartziokas K, Papaioannou AI, Loukides S, et al. Serum uric acid as a predictor of mortality and future exacerbations of COPD. Eur Respir J. 2014;43(1):43-53. doi:10.1183/09031936.00209212

42. Bathoorn E, Groenhof F, Hendrix R, et al. Real-life data on antibiotic prescription and sputum culture diagnostics in acute exacerbations of COPD in primary care. Int $J$ Chron Obstruct Pulmon Dis. 2017;12:285-290. doi:10.2147/COPD.S120510

43. Chang C, Yao W. Time course of inflammation resolution in patients with frequent exacerbations of chronic obstructive pulmonary disease. Med Sci Monit. 2014;20:311-320. doi:10.12659/MSM.889828
44. Cushen B, McCormack N, Hennigan K, Sulaiman I, Costello RW, Deering B. A pilot study to monitor changes in spirometry and lung volume, following an exacerbation of Chronic Obstructive Pulmonary Disease (COPD), as part of a supported discharge program. Respir Med. 2016;119:55-62. doi:10.1016/j. rmed.2016.08.019

45. Hu WP, Lhamo T, Liu D, et al. Development of a nomogram to predict the risk of 30-day re-exacerbation for patients hospitalized for acute exacerbation of chronic obstructive pulmonary disease. COPD. 2019;16(2):160-167. doi:10.1080/15412555.2019.1606187

46. Johannesdottir SA, Christiansen CF, Johansen MB, et al. Hospitalization with acute exacerbation of chronic obstructive pulmonary disease and associated health resource utilization: a population-based Danish cohort study. J Med Econ. 2013;16 (7):897-906. doi:10.3111/13696998.2013.800525

47. Liu D, Peng SH, Zhang J, Bai SH, Liu HX, Qu JM. Prediction of short term re-exacerbation in patients with acute exacerbation of chronic obstructive pulmonary disease. Int $J$ Chron Obstruct Pulmon Dis. 2015;10:1265-1273. doi:10.2147/COPD.S83378

48. Perera WR, Hurst JR, Wilkinson TM, et al. Inflammatory changes, recovery and recurrence at COPD exacerbation. Eur Respir J. 2007;29(3):527-534. doi:10.1183/09031936.00092506

49. Wang L, Zang XY, Zhang Q, Liu SY, Shen YH, Zhao Y. Study on factors influencing recrudescent time of postdischarge patients with chronic obstructive pulmonary disease. J Clin Nurs. 2012;21(1112):1507-1514. doi:10.1111/j.1365-2702.2011.03899.x

50. Yount SE, Atwood C, Donohue J, et al. Responsiveness of PROMIS(R) to change in chronic obstructive pulmonary disease. J Patient Rep Outcomes. 2019;3(1):65. doi:10.1186/s41687-019-0155-9

51. Beauchesne MF, Julien M, Julien LA, et al. Antibiotics used in the ambulatory management of acute COPD exacerbations. Int $J$ Chron Obstruct Pulmon Dis. 2008;3(2):319-322. doi:10.2147/COPD.S2624

52. Crisafulli E, Torres A, Huerta A, et al. Predicting in-hospital treatment failure $(</=7$ days) in patients with COPD exacerbation using antibiotics and systemic steroids. COPD. 2016;13(1):82-92. doi:10.3109/15412555.2015.1057276

53. Dewan NA, Rafique S, Kanwar B, et al. Acute exacerbation of COPD: factors associated with poor treatment outcome. Chest. 2000;117(3):662-671. doi:10.1378/chest.117.3.662

54. Garcia-Sidro P, Naval E, Martinez Rivera C, et al. The CAT (COPD Assessment Test) questionnaire as a predictor of the evolution of severe COPD exacerbations. Respir Med. 2015;109(12):1546-1552. doi:10.1016/j.rmed.2015.10.011

55. Gaude GS, Rajesh BP, Chaudhury A, Hattiholi J. Outcomes associated with acute exacerbations of chronic obstructive pulmonary disorder requiring hospitalization. Lung India. 2015;32(5):465-472. doi:10.4103/0970-2113.164150

56. Lindenauer PK, Pekow PS, Lahti MC, Lee Y, Benjamin EM, Rothberg MB. Association of corticosteroid dose and route of administration with risk of treatment failure in acute exacerbation of chronic obstructive pulmonary disease. JAMA. 2010;303 (23):2359-2367. doi:10.1001/jama.2010.796

57. Miravitlles M, Llor C, Naberan K, Cots JM, Molina J; EFEMAP study group. Group Es. Variables associated with recovery from acute exacerbations of chronic bronchitis and chronic obstructive pulmonary disease. Respir Med. 2005;99(8):955-965. doi:10.1016/j.rmed.2005.01.013

58. Miravitlles M, Izquierdo I, Herrejon A, et al. COPD severity score as a predictor of failure in exacerbations of COPD. The ESFERA study. Respir Med. 2011;105(5):740-747. doi:10.1016/j.rmed.2010.12.020

59. Miravitlles M, Garcia-Polo C, Domenech A, Villegas G, Conget F, de la Roza C. Clinical outcomes and cost analysis of exacerbations in chronic obstructive pulmonary disease. Lung. 2013;191(5):523-530. doi:10.1007/s00408-013-9487-z 
60. Planquette B, Peron J, Dubuisson E, et al. Antibiotics against Pseudomonas aeruginosa for COPD exacerbation in ICU: a 10-year retrospective study. Int $J$ Chron Obstruct Pulmon Dis. 2015;10:379-388. doi:10.2147/COPD.S71413

61. Rothberg MB, Pekow PS, Lahti M, Brody O, Skiest DJ, Lindenauer PK. Comparative effectiveness of macrolides and quinolones for patients hospitalized with acute exacerbations of chronic obstructive pulmonary disease (AECOPD). J Hosp Med. 2010;5 (5):261-267. doi:10.1002/jhm.628

62. Rothberg MB, Pekow PS, Lahti M, Brody O, Skiest DJ, Lindenauer PK. Antibiotic therapy and treatment failure in patients hospitalized for acute exacerbations of chronic obstructive pulmonary disease. JAMA. 2010;303(20):2035-2042. doi:10.1001/jama.2010.672

63. Anzueto A, Miravitlles M, Ewig S, Legnani D, Heldner S, Stauch K. Identifying patients at risk of late recovery ( $>/=8$ days) from acute exacerbation of chronic bronchitis and COPD. Respir Med. 2012;106 (9):1258-1267. doi:10.1016/j.rmed.2012.06.002

64. Aaron SD, Whitmore GA, Hurst JR, Ramsay T, Wedzicha JA. Time course and pattern of COPD exacerbation onset. Thorax. 2012;67 (3):238-243. doi:10.1136/thoraxjnl-2011-200768

65. Wilkinson TM, Donaldson GC, Hurst JR, Seemungal TA, Wedzicha JA. Early therapy improves outcomes of exacerbations of chronic obstructive pulmonary disease. Am J Respir Crit Care Med. 2004;169(12):1298-1303. doi:10.1164/rccm.200310-1443OC

66. Liang Y, Chen Y, Wu R, et al. Chronic bronchitis is associated with severe exacerbation and prolonged recovery period in Chinese patients with COPD: a multicenter cross-sectional study. J Thorac Dis. 2017;9(12):5120-5130. doi:10.21037/jtd.2017.11.54

67. Mackay AJ, Donaldson GC, Patel AR, Singh R, Kowlessar B, Wedzicha JA. Detection and severity grading of COPD exacerbations using the exacerbations of chronic pulmonary disease tool (EXACT). Eur Respir J. 2014;43(3):735-744. doi:10.1183/09031936.00110913

68. Miravitlles M, Anzueto A, Ewig S, Legnani D, Stauch K. Characterisation of exacerbations of chronic bronchitis and COPD in Europe: the GIANT study. Ther Adv Respir Dis. 2009;3 (6):267-277. doi:10.1177/1753465809352791

69. Farias R, Sedeno M, Beaucage D, et al. Innovating the treatment of COPD exacerbations: a phone interactive telesystem to increase COPD Action Plan adherence. BMJ Open Respir Res. 2019;6(1): e000379. doi:10.1136/bmjresp-2018-000379
70. Tsai CL, Rowe BH, Camargo CA Jr. Factors associated with short-term recovery of health status among emergency department patients with acute exacerbations of chronic obstructive pulmonary disease. Qual Life Res. 2009;18(2):191-199. doi:10.1007/s11136008-9437-z

71. Seemungal TA, Donaldson GC, Bhowmik A, Jeffries DJ, Wedzicha JA. Time course and recovery of exacerbations in patients with chronic obstructive pulmonary disease. Am J Respir Crit Care Med. 2000;161(5):1608-1613. doi:10.1164/ ajrccm.161.5.9908022

72. Donaldson GC, Law M, Kowlessar B, et al. Impact of prolonged exacerbation recovery in chronic obstructive pulmonary disease. $\mathrm{Am}$ J Respir Crit Care Med. 2015;192(8):943-950. doi:10.1164/ rccm.201412-22690C

73. Aaron SD, Donaldson GC, Whitmore GA, Hurst JR, Ramsay T, Wedzicha JA. Time course and pattern of COPD exacerbation onset. Thorax. 2012;67(3):238-243. doi:10.1136/thoraxjnl-2011200768

74. McIlvennan CK, Eapen ZJ, Allen LA. Hospital readmissions reduction program. Circulation. 2015;131(20):1796-1803. doi:10.1161/ CIRCULATIONAHA.114.010270

75. Trappenburg JC, Schaap D, Monninkhof EM, et al. How do COPD patients respond to exacerbations? BMC Pulm Med. 2011;11:43. doi:10.1186/1471-2466-11-43

76. Agusti A, Calverley PM, Decramer M, Stockley RA, Wedzicha JA. Prevention of exacerbations in chronic obstructive pulmonary disease: knowns and unknowns. COPD. 2014;1(2):166-184. doi:10.15326/jcopdf.1.2.2014.0134

77. Wedzicha JA. Exacerbations: etiology and pathophysiologic mechanisms. Chest. 2002;121(5, Supplement):136S-141S. doi:10.1378/chest.121.5_suppl.136S

78. Meeraus W, Mullerova H, Tabberer M, Fahy WA, Benson VS. Systematic review of COPD re-exacerbation and exacerbation treatment failure definitions used in randomised controlled trials (RCTs). Eur Respirat J. 2018;52(suppl 62):PA1171.

\section{Publish your work in this journal}

The International Journal of COPD is an international, peer-reviewed journal of therapeutics and pharmacology focusing on concise rapid reporting of clinical studies and reviews in COPD. Special focus is given to the pathophysiological processes underlying the disease, intervention programs, patient focused education, and self management protocols. This journal is indexed on PubMed Central, MedLine and CAS. The manuscript management system is completely online and includes a very quick and fair peer-review system, which is all easy to use. Visit http://www.dovepress.com/testimonials.php to read real quotes from published authors. 